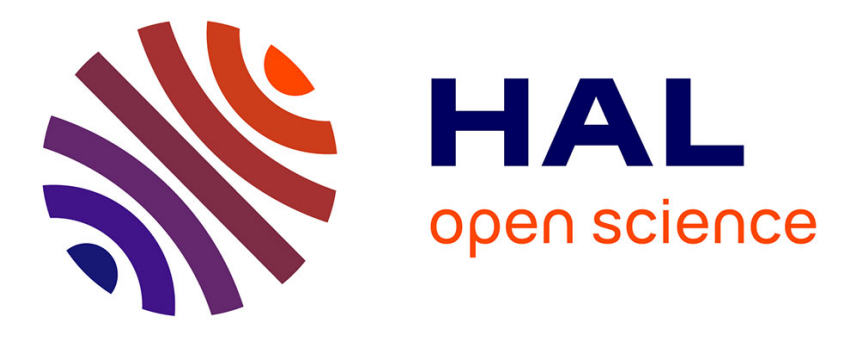

\title{
Réalisation d'un spectromètre d'absorption " picoseconde " monofaisceau. Application à la dynamique des états excités des molécules organiques
}

\author{
C. Rullière, A. Declemy, Ph. Pée
}

\section{- To cite this version:}

C. Rullière, A. Declemy, Ph. Pée. Réalisation d'un spectromètre d'absorption " picoseconde " monofaisceau. Application à la dynamique des états excités des molécules organiques. Revue de Physique Appliquée, 1983, 18 (6), pp.347-354. 10.1051/rphysap:01983001806034700 . jpa-00245101

HAL Id: jpa-00245101

https://hal.science/jpa-00245101

Submitted on 1 Jan 1983

HAL is a multi-disciplinary open access archive for the deposit and dissemination of scientific research documents, whether they are published or not. The documents may come from teaching and research institutions in France or abroad, or from public or private research centers.
L'archive ouverte pluridisciplinaire HAL, est destinée au dépôt et à la diffusion de documents scientifiques de niveau recherche, publiés ou non, émanant des établissements d'enseignement et de recherche français ou étrangers, des laboratoires publics ou privés. 


\title{
Réalisation d'un spectromètre d'absorption « picoseconde " monofaisceau. Application à la dynamique des états excités des molécules organiques
}

\author{
C. Rullière, A. Declemy et Ph. Pée \\ Centre de Physique Moléculaire Optique et Hertzienne (*), Université de Bordeaux I, 33405 Talence Cedex, France
}

(Reçu le 7 février 1983, accepté le 18 mars 1983)

\begin{abstract}
Résumé. - Un spectromètre d'absorption résolue en temps et entièrement automatisé a été construit. Il permet d'obtenir des spectres d'absorption et de gain d'états excités de durée de vie $\tau>10$ ps. Ce spectromètre utilise une impulsion lumineuse d'une durée de 25 ps délivrée par un laser $\mathrm{YAG} / \mathrm{Nd}^{3+}$ à modes bloqués. Le faisceau d'analyse est obtenu en focalisant cette impulsion dans une cellule contenant de l'eau lourde $\mathrm{D}_{2} \mathrm{O}$ (création d'un continuum). Nous avons déterminé des conditions expérimentales pour lesquelles la distribution spectrale de ce faisceau ne varie pas. Dans ces conditions, le même faisceau sert à la fois pour l'analyse et la référence et permet d'utiliser un détecteur monodimensionnel constitué par une barrette de photodiodes. On tire ainsi parti des avantages que présente ce type de détecteur (dynamique de réponse importante, robustesse, faible coût) par rapport aux tubes vidicon plus souvent utilisés. La densité optique minimale mesurable couramment avec notre système est de 0,03 et peut même atteindre la valeur de 0,01 moyennant quelques précautions. Le spectre d'absorption d'une espèce excitée à courte durée de vie dans la gamme spectrale $25000-10000 \mathrm{~cm}^{-1}$ est ainsi obtenu en quelques minutes. Quelques exemples d'application (mesure de durée de vie, spectre d'absorption, cinétiques de gain) sont présentés.
\end{abstract}

\begin{abstract}
Picosecond absorption spectrometer has been built. Mode locked YAG/Nd ${ }^{3+}$ laser is used both as an excitation source of the sample to be studied and as a tool in the production of the picosecond light continuum probe beam. High reproducibility of the spectral shape and distribution in regard of his energy allows to use only one probe beam for reference and analysing light. As a consequence linear photodiode array is used as a detector associated to the Tracor Northern DARSS system, allowing complete automatization of the experimental set-up. We take advantage of the photodiode array (high dynamic range, low cost, fiability and easy upkeep) over the vidicon target properties. With our system, optical density of 0.03 are measured as a routine and of 0.01 with particular emphasize. Absorption spectrum of excited species in the spectral range $25000-10000 \mathrm{~cm}^{-1}$ are obtained in three minutes. Some applications are presented such as simultaneous study of absorption and gain spectrum, short decay time measurements and formation constant kinetic on a picosecond time scale.
\end{abstract}

\section{Introduction.}

Les molécules polyatomiques comprenant un très grand nombre d'atomes (jusqu'à 100 atomes) sont au cœur de nombreux mécanismes biologiques tels que les processus de la vision, de la photosynthèse et plus généralement de nombreux mécanismes photophysiques et photochimiques. Ce sont les états excités singulets et triplets de ces molécules qui sont impliqués dans ces mécanismes. La connaissance des caractéristiques photophysiques de ces états (spectres d'absorption, conformation, durée de vie) et leur évolution temporelle est essentielle à la compréhension de ces divers mécanismes. Or la durée de vie de ces états est généralement très courte et se situe dans une gamme

(*) Laboratoire associé au C.N.R.S. $n^{\circ} 283$. temporelle comprise entre quelques femtosecondes et quelques nanosecondes. L'étude de ces états excités nécessite donc l'utilisation de spectromètres d'absorption fonctionnant dans ces échelles de temps très courts.

La réalisation de tels spectromètres a été décrite dans la littérature $[1,3]$ et nous rappellerons brièvement leur principe dans la suite de cet article. Retenons cependant que ces spectrophotomètres utilisent généralement deux faisceaux, l'un d'analyse et l'autre de référence, générés par un laser pulsé. Le faisceau d'analyse traverse une partie de l'échantillon préalablement excité par une impulsion laser brève, et voit sa distribution spectrale perturbée par la présence de molécules à l'état excité. Le faisceau de référence traverse une partie de l'échantillon non excité et sa distribution spectrale n'est donc pas perturbée par la 
présence de molécules à l'état excité. La comparaison de la distribution spectrale des deux faisceaux permet d'obtenir le spectre d'absorption des états excités et d'étudier leur évolution temporelle. Cependant cette comparaison nécessite une mémorisation rapide des distributions spectrales à chaque tir laser. Cette mémorisation est généralement obtenue en dirigeant ces deux faisceaux (préalablement dispersés spectralement par passage dans un spectrographe) sur la surface sensible d'un tube vidicon. Cette surface sensible, constituée d'une mosaïque de diodes au silicium, est divisée en deux parties recevant respectivement le faisceau d'analyse et le faisceau de référence.

Le tube vidicon est alors programmé pour lire chaque partie de la surface sensible et mesurer ainsi la distribution spectrale de chacun des faisceaux. Cette méthode présente cependant quelques inconvénients. En effet la division en deux parties de la surface sensible du tube vidicon entraîne une diminution importante de la dynamique de mesure du tube vidicon, et la variation de distribution spectrale d'intensité est alors limitée à une faible gamme spectrale. De plus il est généralement difficile d'obtenir une distribution spectrale identique pour les deux faisceaux d'analyse [2], ce qui implique des corrections à apporter à chaque mesure.

Dans cet article nous montrons qu'il est possible de réaliser un spectromètre d'absorption travaillant à l'échelle de la picoseconde en utilisant un même faisceau à la fois pour l'analyse et la référence. Ceci permet d'employer un détecteur monodimensionnel constitué par une barrette de photodiodes et de disposer ainsi des nombreux avantages que présentent ce type de détecteur par rapport aux tubes vidicon (gamme dynamique, souplesse d'emploi, stabilité, moindre fragilité, faible maintenance, coût faible).

\section{Description de l'appareil.}

2.1 RAPPEL DU PRINCIPE DE FONCTIONNEMENT. A l'aide d'une impulsion brève de lumière, on porte brusquement un nombre de molécules $N_{\mathrm{S}^{*}}$ dans un état excité $S^{*}$ de durée de vie $\tau$. Cette population que nous désignerons par $N_{\mathrm{S}^{*}}(t)$ va alors évoluer au cours du temps, entraînant des variations temporelles des propriétés spectrales du milieu excité. Ce sont ces variations que le dispositif expérimental doit permettre d'étudier. Cette étude est faite en envoyant à un instant donné $t$ une impulsion lumineuse appelée impulsion de sonde (de durée $\Delta t$ courte devant $\tau$ ) à travers l'échantillon excité au temps $t=0$. Si on désigne alors par $\sigma_{\mathrm{a}}(v)$ la section efficace d'absorption de l'état $\mathrm{S}^{*}$ à la fréquence $v$ et par $I_{0}(v)$ et $I(v, t)$ la distribution spectrale d'intensité de l'impulsion sonde, avant et après traversée de l'échantillon, la loi de Beer-Lambert conduit à l'égalité suivante :

$$
I(v, t)=I_{0}(v) \times \mathrm{e}^{-\left\{\sigma_{\mathbf{a}}(v) N_{\mathbf{s}}^{*}(t) l\right\}}
$$

(où $l$ est la longueur du parcours de l'impulsion lumi- neuse dans l'échantillon), égalité que l'on peut écrire sous la forme suivante :

$D O(v, t)=\log \frac{I_{0}(v)}{I(v, t)}=\sigma_{\mathrm{a}}(v) N_{\mathrm{S}^{*}}(t) l \times \log e$,

(où $D O$ est appelée densité optique).

La mesure des variations de la densité optique en fonction de la fréquence $v$ et du temps $t$ permet alors d'obtenir le spectre d'absorption de l'état $S^{*}$ (décrit par $\left.\sigma_{\mathrm{a}}(v)\right)$ à un instant donné, ainsi que les variations temporelles de $N_{\mathrm{S}^{*}}(t)$ à une fréquence donnée $v$. La mesure de la quantité $D O(v, t)$ consiste donc à mesurer la variation de distribution spectrale d'un faisceau de lumière traversant un échantillon en présence d'excitation $(I(v, t))$ et en absence d'excitation $I_{0}(v)$.

2.2 SOURCE LASER UTILISÉE ET MONTAGE. - La source utilisée est un laser $\mathrm{YAG} / \mathrm{Nd}^{3+}$ à modes bloqués délivrant une impulsion lumineuse unique d'une durée d'environ 25 ps à $1,06 \mu$. Cette impulsion est alors divisée en deux faisceaux lumineux par l'intermédiaire d'un amplificateur à double passage (cf. Fig. 1).

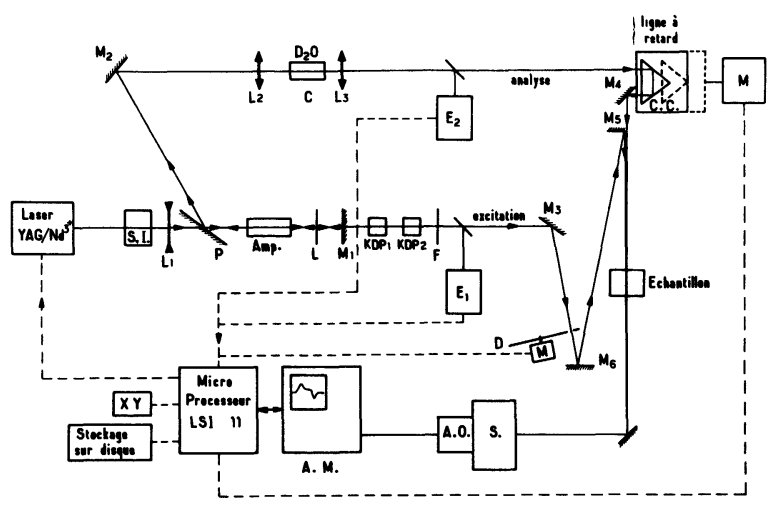

Fig. 1. - Montage expérimental. S.I. : sélecteur d'impulsion. $L_{1}$ : lentille divergente. $P$ : polariseur diélectrique. Amp : amplificateur. $L$ : lame $1 / 4 \lambda$ à $1,06 \mu$. $M_{1}$ : miroir semi-transparent à $1,06 \mu R=20 \%$. F : filtre. $\mathbf{M}_{2}, \mathbf{M}_{3}, \mathbf{M}_{4}$, $\mathbf{M}_{5}, \mathbf{M}_{6}$ : miroirs totalement réfléchissants. $\mathrm{C}$ : cellule d'eau lourde. C.C. : coin de cube. S : spectrographe. A.O. : analyseur optique. A.M. : analyseur multicanal. $M$ : moteur. $\mathrm{D}$ : disque.

[Experimental set-up. S.I. : pulse selector. $\mathrm{L}_{1}$ : divergent lens. $\mathbf{P}$ : dielectric polarizer. Amp : amplifier. $\mathrm{L}$ : quarterwave plate at $1.06 \mu . \mathrm{M}_{1}$ : partially reflecting mirror at $1.06 \mu$ $R=20 \%$. F : filter. $\mathbf{M}_{2}, \mathbf{M}_{3}, \mathbf{M}_{4}, \mathbf{M}_{5}, \mathbf{M}_{6}$ : totally reflecting mirrors. $\mathrm{C}$ : cell containing $\mathrm{D}_{2}$ O. C.C. : cube corner. $\mathrm{S}$ : spectrograph. A.O. : optical analyser. A.M. : multichannel analyser. $\mathbf{M}$ : motor. $\mathrm{D}$ : disk.]

La première partie du faisceau dont l'énergie est d'environ $4 \mathrm{~mJ}$ sert à la création de fréquences multiples de $1,06 \mu$ par passage à travers des cristaux de KDP. On obtient ainsi des énergies de l'ordre de $1 \mathrm{~mJ}$ à $0,53 \mu, 300 \mu \mathrm{J}$ à $0,353 \mu$ et quelques dizaines de $\mu \mathrm{J}$ à $0,265 \mu$. Cette partie du faisceau sert à exciter 
l'échantillon et donc à porter des molécules dans l'état S*.

La deuxième partie du faisceau qui a traversé deux fois l'amplificateur (cf. Fig. 1) a une énergie d'environ $30 \mathrm{~mJ}$. Cette partie sert à générer le faisceau sonde de distribution spectrale $I_{0}(v)$. Ce faisceau sonde est obtenu en focalisant l'impulsion dans une cuve contenant de l'eau lourde $\left(\mathrm{D}_{2} \mathrm{O}\right)$ (cf. Fig. 1). A la suite des travaux d'Alfano et Shapiro [4] on sait en effet que la focalisation d'une impulsion lumineuse brève et intense dans différents liquides $\left(\mathrm{CCl}_{4}, \mathrm{H}_{2} \mathrm{O}, \mathrm{D}_{2} \mathrm{O}\right)$ conduit à la création d'un faisceau de lumière dont les caractéristiques temporelles et géométriques sont identiques à l'impulsion génératrice (directivité et durée de cette impulsion) mais dont les caractéristiques spectrales sont complètement modifiées. On parle dans la littérature de "continuum de lumière " car la distribution spectrale de ce faisceau peut s'étendre de 35000 à $5000 \mathrm{~cm}^{-1}$. Ces diverses caractéristiques en font donc un faisceau sonde largement utilisé dans ce genre d'expérience. Dans notre cas, ce faisceau est envoyé sur un coin de cube monté sur une platine mobile, puis dirigé vers l'échantillon (cf. Fig. 1). L'ensemble coin de cube-platine mobile sert de ligne à retard optique et permet ainsi de faire varier l'intervalle de temps entre l'instant d'arrivée du faisceau excitateur sur l'échantillon et l'instant d'arrivée du faisceau sonde (continuum de lumière) sur l'échantillon. Dans notre montage, la ligne à retard optique permet de faire varier cet intervalle de temps entre 0 et 500 ps. Lorsqu'un intervalle de temps plus grand est nécessaire, on modifie le trajet optique du faisceau excitateur.

2.3 PrinciPe De LA MesURe. - Comme nous l'avons montré précédemment, le principe de fonctionnement du spectromètre décrit dans cet article nécessite de mesurer la distribution spectrale du faisceau sonde en absence et en présence du faisceau d'excitation dans l'échantillon.

Dans notre montage expérimental, ces mesures sont effectuées entièrement automatiquement à l'aide d'un microprocesseur LSI 11 associé à un analyseur optique multicanal (Tracor Northern 1710 - système DARSS).

La distribution spectrale du faisceau sonde est mesurée à l'aide d'une barrette de photodiodes (512 diodes) placée dans le plan focal d'un spectrographe. La température des diodes est stabilisée à $5^{\circ} \mathrm{C}$ afin de minimiser leur variation d'efficacité quantique et de réponse.

L'absence ou la présence du faisceau excitateur dans l'échantillon est contrôlée à l'aide d'un disque tournant, percé de trous, placé devant ce faisceau. Le disque est entraîné par un moteur pas à pas commandé par le microprocesseur (cf. Fig. 1).

L'intervalle de temps $\Delta t$ entre l'instant d'arrivée $\mathrm{du}$ faisceau excitateur sur l'échantillon et l'instant d'arrivée du faisceau sonde est aussi contrôlé par le microprocesseur qui commande un moteur pas à pas dont l'axe est relié à la platine mobile (cf. Fig. 1).

Comme nous le verrons dans la suite de cet article, le principe de la mesure implique que deux conditions expérimentales soient remplies. D'une part l'énergie du faisceau excitateur doit rester identique au cours de l'expérience et d'autre part la distribution spectrale $I_{0}(v, t)$ du continuum doit rester constante, ou tout au moins les différents profils spectraux $I_{0}(v)$ doivent rester homothétiques d'un tir laser à l'autre (cf. appendice I). Ces deux conditions expérimentales sont vérifiées à l'aide de deux échantillonneurs $E_{1}$ et $E_{2}$ (cf. Fig. 1).

Le premier échantillonneur $\mathrm{E}_{1}$ mesure une quantité $I_{\mathrm{E}_{1}}$ proportionnelle à l'énergie d'excitation. Cette quantité est lue puis comparée, à l'aide du microprocesseur, à une valeur témoin donnée par l'opérateur au début de la manipulation. Si la valeur lue s'éloigne trop de la valeur témoin, le tir laser n'est pas pris en compte. Un nouveau tir laser est alors déclenché et testé de façon identique. Ce processus a lieu automatiquement autant de fois qu'il est nécessaire pour obtenir une valeur lue identique (à quelques $\%$ près) à la valeur témoin. L'échantillonneur $E_{2}$ procède de la même façon pour le faisceau sonde et mesure une valeur $I_{\mathrm{E}_{2}}$ proportionnelle à la quantité $\int_{0}^{\infty} I_{0}(v) \times$ $K(v) \mathrm{d} v$ (où $K(v)$ désigne la sensibilité spectrale de l'échantillonneur $E_{2}$ ). On sait que la distribution spectrale $I_{0}(v)$ du faisceau sonde créé varie fortement avec l'intensité de l'impulsion laser à $1,06 \mu[5,6]$. Il en est donc de même de : $\int_{0}^{\infty} I_{0}(v) K(v) \mathrm{d} v$ lue par
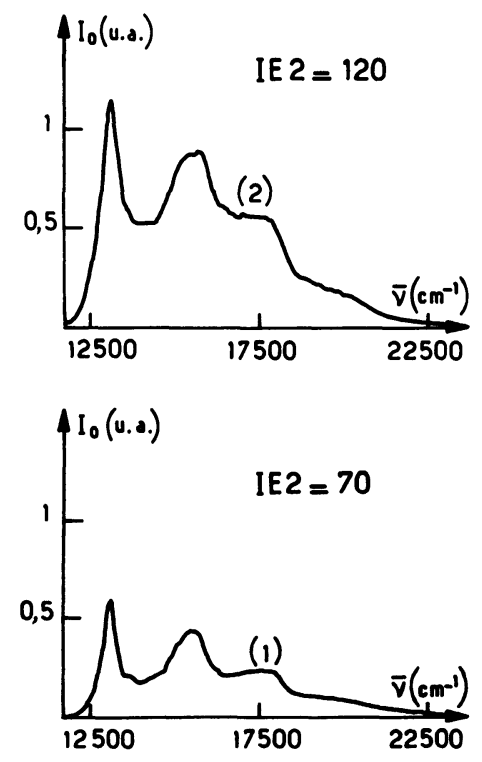

Fig. 2. - Distribution spectrale du faisceau sonde pour deux valeurs de $I_{\mathrm{E}_{2}}$ situées en dehors de la plage de reproductibilité (cf. texte). (1) $I_{\mathrm{E}_{2}}=70$, (2) $I_{\mathrm{E}_{2}}=120$. $I_{\mathrm{E}_{2}}$ est en unités arbitraires.

[Probe beam spectral distribution for two $I_{\mathrm{E}_{2}}$ values outside of usable range (cf. text); (1) $I_{\mathrm{E}_{2}}=70$; (2) $I_{\mathrm{E}_{2}}=120$. $\left(I_{E_{2}}\right.$ in arbitrary units):] 
l'échantillonneur $\mathrm{E}_{2}$. La figure 2 donne à cet égard quelques exemples frappants. Nous avons cependant trouvé une corrélation entre les valeurs $I_{\mathrm{E}_{2}}$ lues par l'échantillonneur $\mathrm{E}_{2}$ et la distribution spectrale $I_{0}(v)$. En particulier nous avons trouvé une plage de valeurs de $I_{\mathrm{E}_{2}}$ pour lesquelles les différents profils spectraux $I_{0}(v)$ restent homothétiques. Cette propriété est illustrée sur la figure 3. Si la valeur $I_{\mathrm{E}_{2}}$ ne se trouve pas dans cette plage, le tir laser n'est pas pris en compte. Un autre tir laser est alors programmé et testé de façon identique par $E_{2}$. Ce processus cesse lorsque la valeur lue de $I_{\mathrm{E}_{2}}$ se trouve dans la plage de valeurs préalablement programmée par l'opérateur.
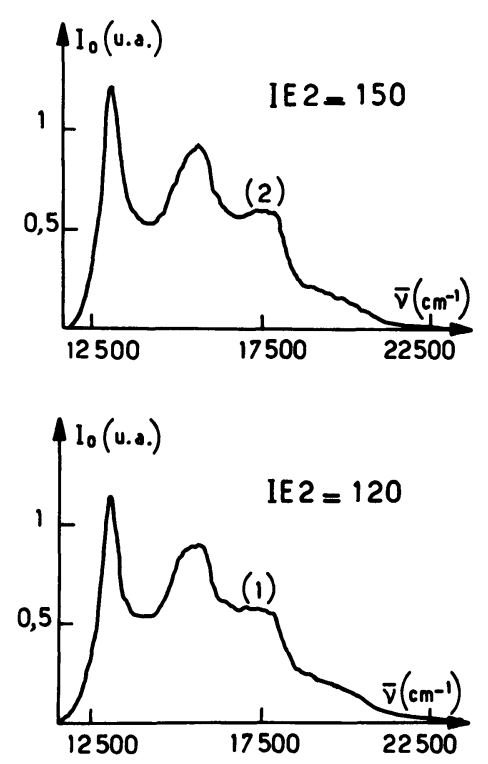

Fig. 3. - Distribution spectrale du faisceau sonde pour des valeurs de $I_{\mathrm{E}_{2}}$ situées à l'intérieur de la plage de reproductibilité (cf. texte). (1) $I_{\mathrm{E}_{2}}=120$, (2) $I_{\mathrm{E}_{2}}=150$. $I_{\mathrm{E}_{2}}$ est en unités arbitraires.

[Probe beam spectral distribution for $I_{\mathrm{E}_{2}}$ values inside the usable range (cf. text); (1) $I_{\mathrm{E}_{2}}=120$; (2) $I_{\mathrm{E}_{2}}=150$. $\left(I_{\mathrm{E}_{2}}\right.$ in arbitrary units).]

La mesure de la quantité $D O(v, t)$ a lieu chronologiquement de la façon suivante. L'opérateur définit tout d'abord le nombre de tirs laser à effectuer, les différentes valeurs de $\Delta t$ souhaitées, ainsi que les plages de valeurs de $I_{E_{1}}$ et $I_{E_{2}}$ à l'intérieur desquelles la reproductibilité de l'expérience est acceptable (valeurs témoins). Ensuite pour une position de la platine mobile correspondant à un temps $t$ donné et pour une position du disque tournant laissant passer le faisceau excitateur, un tir laser est exécuté. Les valeurs de $I_{E_{1}}$ et $I_{E_{2}}$ ainsi que la distribution spectrale $I(v, t)$ sont mesurées. Si les valeurs lues $I_{\mathrm{E}_{1}}$ et $I_{\mathrm{E}_{2}}$ ne satisfont pas aux conditions de reproductibilité, le tir laser n'est pas pris en compte et un autre tir laser est exécuté. Lorsque les valeurs de $I_{\mathrm{E}_{1}}$ et $I_{\mathrm{E}_{2}}$ correspondent aux valeurs témoins, le tir laser est pris en compte et les valeurs $I(v, t)$ sont stockées dans une mémoire (mémoire A) de l'analyseur multicanal. Le moteur du disque tournant est alors commandé de façon à bloquer le faisceau excitateur et à mesurer $I_{0}(v)$. Un tir laser est exécuté et comme précédemment, lorsque les valeurs $I_{E_{1}}$ et $I_{E_{2}}$ satisfont aux conditions de reproductibilité, la quantité $I_{0}(v)$ est stockée dans une autre mémoire (mémoire B) de l'analyseur multicanal. Le processus de mesure de $I(v, t)$ et $I_{0}(v)$ ainsi décrit est reconduit jusqu'à ce que le nombre de tirs laser pris en compte atteigne la valeur définie par l'opérateur au début de l'expérience. Le nombre de tirs laser programmé dans notre cas est généralement de 25 . Pour des expériences nécessitant plus de soins (faible densité optique à mesurer) il est de 100 tirs. Lorsque le nombre de tirs laser souhaité est atteint, on calcule automatiquement à l'aide du microprocesseur la quantité $D O(v, t)=\log \left\{I_{0}(v) / I(v, t)\right\}$ et le résultat est stocké sur disque. Ensuite le moteur de la platine mobile est commandé de façon à ajuster le temps $t$ à une valeur telle que définie par l'opérateur et le même cycle reprend jusqu'à ce que les mesures aient été effectuées pour toutes les valeurs de $t$ souhaitées. A la fin de l'expérience on a donc stocké sur disque toutes les valeurs de $D O(v, t)$ pour l'intervalle spectral couvert par la barrette de photodiodes et pour différents temps $t$. Le dépouillement de ces résultats peut alors commencer. Avant d'illustrer par quelques résultats d'expérience les applications possibles de ce dispositif expérimental, nous allons présenter ses performances.

2.4 Performances du Système. - La densité optique minimum détectable avec notre système est de 0,03 en accumulant 25 tirs laser et de 0,01 en accumulant 100 tirs laser. Les figures $4 a$ et $4 b$ montrent les résultats obtenus en utilisant un échantillon de densité optique nulle. Cette densité optique nulle est créée en bloquant le disque tournant dans une même position afin de rendre $I_{0}(v)$ égal à $I(v, t)$.

On peut rendre ce système encore plus performant en augmentant le nombre de tirs laser accumulés. En fait pour manipuler confortablement nous nous limitons à 25 tirs lorsque la valeur maximale de $D O(v, t)$ est de l'ordre de 1 , et 100 tirs lorsque cette valeur est plus faible et que l'on désire une plus grande précision.

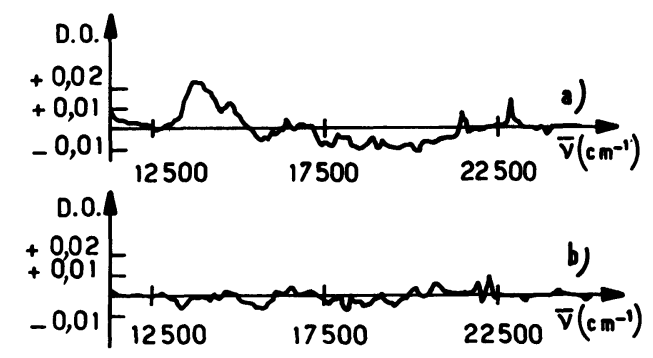

Fig. 4. - Densité optique minimum détectable en fonction de la fréquence v. a) 25 tirs laser pris en compte; b) 100 tirs laser pris en compte.

[Smallest detectable optical density as a function of frequency v. a) 25 laser shots; b) 100 laser shots.] 
Le domaine spectral couvert s'étend de 10000 à $25000 \mathrm{~cm}^{-1}$. Ceci signifie que nous pouvons obtenir pour chaque série de mesure le spectre d'absorption d'une espèce excitée entre 10000 et $25000 \mathrm{~cm}^{-1}$. La résolution spectrale dépend de la dispersion du spectrographe utilisée et de la distribution spatiale des photodiodes. Dans notre cas elle est de $20 \mathrm{~cm}^{-1}$ à la fréquence de $20000 \mathrm{~cm}^{-1}$.

Le temps nécessaire pour obtenir un spectre à un instant $t$ donné est de l'ordre de $3 \mathrm{~min}$. lorsque l'on désire faire l'expérience en accumulant 25 tirs. La cadence de tirs du laser est en effet de $1 \mathrm{~Hz}$ et le système prend en compte en moyenne un tir laser sur trois.

\section{Quelques exemples d'application.}

3.1 Mesures De SPECTRES D'ABSORPTION. - Les figures 5 et 6 montrent les spectres d'absorption $S_{1}^{*} \rightarrow S_{n}^{*}$ du premier état singulet excité de deux composés organiques en solution : le perylène (Fig. 5) et le B.B.O. ou di-biphenylyl oxazole (Fig. 6). Nous avons choisi ces deux composés pour deux raisons. D'une part la durée de vie du premier état singulet excité de ces composés est courte $(5 \mathrm{~ns}$ [7] pour le perylène et $1,15 \mathrm{~ns}$ [7] pour le B.B.O.) ce qui permet donc de tester notre dispositif sur des espèces excitées à courte durée de vie. D'autre part leur spectre $S_{1}^{*} \rightarrow$ $\mathrm{S}_{n}^{*}$ a été mesuré par d'autres méthodes et il est intéressant de comparer les résultats des différentes méthodes.

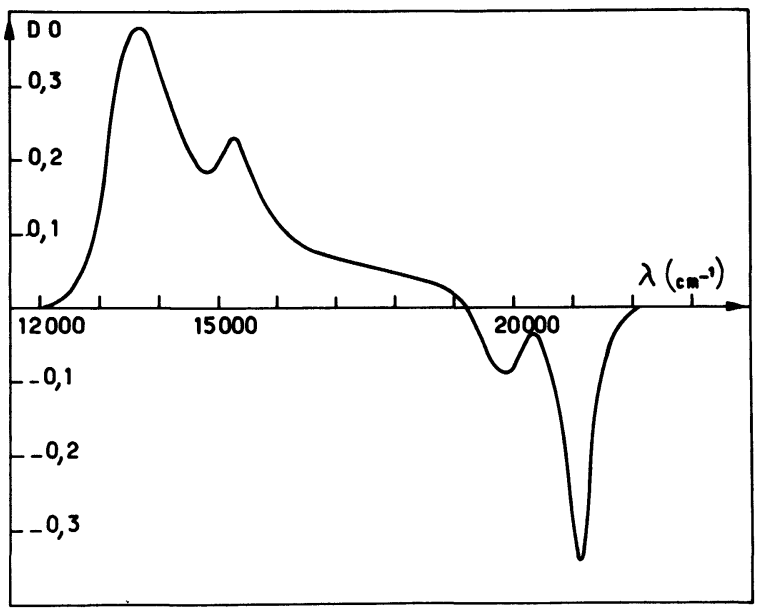

Fig. 5. - Spectre $S_{1}^{*} \rightarrow S_{n}^{*}$ du perylène en solution dans le cyclohexane. $C=10^{-4} \mathrm{M} / 1, T=20^{\circ} \mathrm{C}$.

$\left[\mathrm{S}_{1}^{*} \rightarrow \mathrm{S}_{n}^{*}\right.$ absorption spectrum of perylene diluted in cyclohexane. $C=10^{-4} \mathrm{M} / \mathrm{l}, T=20^{\circ} \mathrm{C}$.]

Le spectre $S_{1}^{*} \rightarrow S_{n}^{*}$ du perylène a été mesuré par Goldschmidt et al. [8] par une méthode de photolyse éclair utilisant un laser à azote pulsé. Ces auteurs avaient obtenu seulement une vingtaine de points pour tracer le spectre $S_{1}^{*} \rightarrow S_{n}^{*}$ ce qui conduit par exemple à une incertitude sur la position du maximum de ce spectre. Avec notre dispositif expérimental nous sommes capables d'obtenir ce spectre avec 512 points. Nous pouvons ainsi sans ambiguité

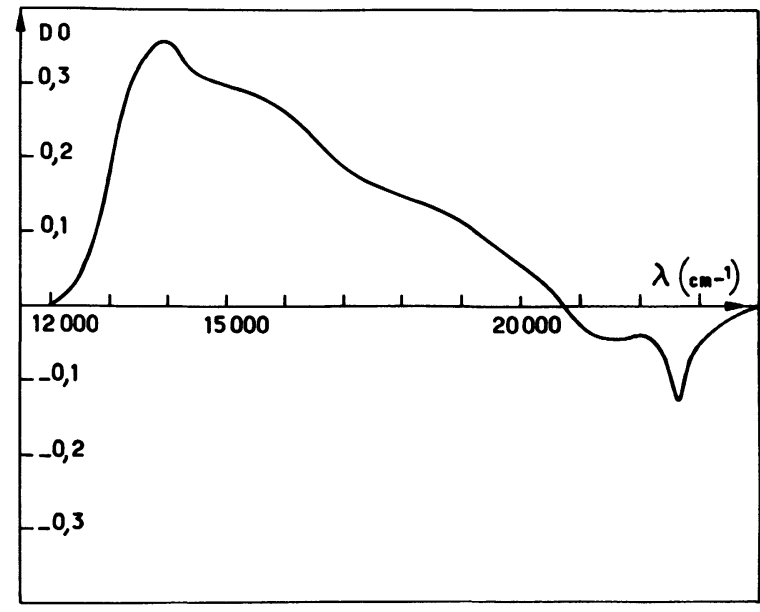

Fig. 6. - Spectre $S_{1}^{*} \rightarrow S_{n}^{*}$ du 2,5 di-biphénylyl-oxazole (B.B.O.) en solution dans le chlorobenzène. $C=10^{-4} \mathrm{M} / \mathrm{l}$, $T=20^{\circ} \mathrm{C}$

[ $\mathrm{S}_{1}^{*} \rightarrow \mathrm{S}_{n}^{*}$ absorption spectrum of 2,5 di-biphenylyl-oxazole (B.B.O.) diluted in chlorobenzene. $C=10^{-4} \mathrm{M} / 1, T=$ $20^{\circ} \mathrm{C}$.]

placer à $13600 \pm 50 \mathrm{~cm}^{-1}$ le maximum du spectre $S_{1}^{*} \rightarrow S_{n}^{*}$. De plus notre spectre met en évidence deux nouvelles bandes d'absorption, l'une à $15200 \mathrm{~cm}^{-1}$ et l'autre vers $17500 \mathrm{~cm}^{-1}$. Ces deux bandes n'avaient pas été observées précédemment [8]. Le spectre $\mathrm{S}_{1}^{*} \rightarrow \mathrm{S}_{n}^{*}$ du B.B.O. avait été mesuré par Fouassier et al. [9] en utilisant un laser à colorant synchrone d'un laser à rubis à modes bloqués. Ces auteurs n'avaient pu obtenir que neuf points entre 20000 et $13000 \mathrm{~cm}^{-1}$ pour tracer le spectre. Avec 512 points entre 12000 et $25000 \mathrm{~cm}^{-1}$, la précision obtenue explique les différences marquées, en particulier sur l'intensité relative des bandes et leur position, entre notre spectre expérimental et celui publié par ces auteurs [9].

Les spectres des figures 5 et 6 montrent aussi qu'il est possible de mesurer le spectre de gain d'espèces excitées. En effet, dans la région spectrale 20000 $24000 \mathrm{~cm}^{-1}$, apparaît une densité optique négative ce qui signifie que le premier état excité singulet $S_{1}^{*}$ de ces composés amplifie la lumière. Ces deux composés (B.B.O. et perylène) sont utilisés comme milieu amplificateur de lasers à colorants $[10,11]$. Notre montage expérimental permet de mesurer le profil spectral de gain en fonction du temps. Connaissant ce profil et le spectre de fluorescence (mesuré par exemple à l'aide d'un spectrofluorimètre), on peut en déduire la section efficace d'absorption $\sigma_{\mathrm{a}}^{s_{i}}(v)$ de l'état $S_{1}^{*}$ à l'intérieur du domaine spectral de fluorescence [12]. Cette mesure est difficile, voir impossible, à mettre en ouvre par d'autres méthodes, l'émission de fluorescence perturbant fortement les résultats. Avec notre montage expérimental il devient aussi facile d'obtenir $\sigma_{\mathrm{a}}^{s^{\mathrm{i}}}(v)$ pour un domaine spectral situé en dehors de la bande de fluorescence qu'à l'intérieur du domaine spectral de fluorescence. 
3.2 MesURe De DURÉE DE VIE. - Pour illustrer cette possibilité de mesure nous avons là aussi choisi le B.B.O. dont la durée de vie $\tau$ du premier état singulet $S_{1}^{*}$ est connue et égale à 1,15 ns [7]. Pour faire cette mesure nous avons enregistré le spectre $\mathrm{S}_{1}^{*} \rightarrow \mathrm{S}_{n}^{*}$ (cf. Fig. 6) de ce composé pour des temps variant de 0 à 500 ps après le temps d'arrivée de l'impulsion excitatrice de la solution.

Sachant que le déclin de l'état $S_{1}^{*}$ du B.B.O. est monoexponentiel [7] nous pouvons écrire l'expression

$$
D O(v, t)=D O(v, 0) \cdot \exp (-t / \tau) .
$$

Un tracé logarithmique, à une fréquence $v$ donnée, de $D O(v, t)$ en fonction du temps donne accès au temps de vie $\tau$. La figure $7 a$ illustre nos résultats. La valeur de $1,28 \mathrm{~ns}$ obtenue pour $\tau$ se compare avantageusement à la valeur de 1,15 ns obtenue par Berlmann [7] par une méthode différente et dans un solvant différent.

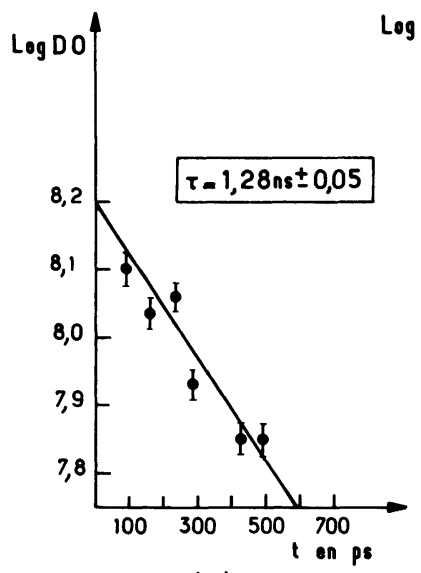

(a)

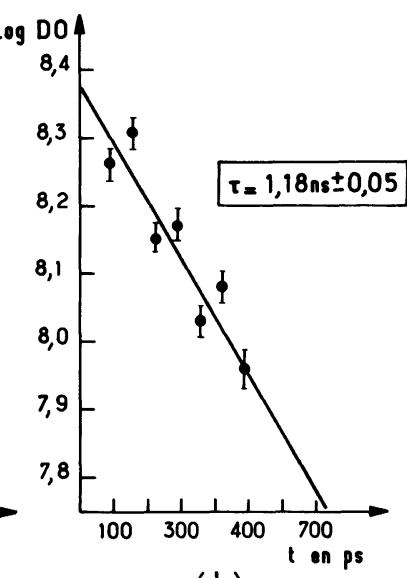

(b)
Fig. 7. - Variations en fonction du temps de la densité optique $D O(v, t)$, à la fréquence $v=22600 \mathrm{~cm}^{-1}$, d'une solution de B.B.O. dans le chlorobenzène. $C=5 \times 10^{-4} \mathrm{M} / \mathrm{l}$, $T=20^{\circ} \mathrm{C}$. a) Sans oxygène dissous dans la solution (dégazage par barbotage d'azote); $b$ ) Avec oxygène dissous dans la solution (saturée d'air).

[Optical density variations $D O(v, t)$ as a function of time for the $v$ frequency $22600 \mathrm{~cm}^{-1}$. B.B.O. solution in chlorobenzene. $C=5 \times 10^{-4} \mathrm{M} / 1, T=20^{\circ} \mathrm{C}$. a) Without diluted oxygen $; b$ ) With diluted oxygen (air saturated).]

On sait d'autre part qu'en présence d'oxygène dissous dans la solution, le temps de vie $\tau$ diminue selon la formule suivante :

$$
'\left(1 / \tau_{\mathrm{O}_{2}}\right)={ }^{\prime}(1 / \tau)+k_{\mathrm{O}_{2}}\left\{\mathrm{O}_{2}\right\},
$$

où

$\tau_{\mathrm{O}_{2}} \quad$ désigne le temps de vie en présence d'oxygène;

$k_{\mathrm{O}_{2}}$ la constante de vitesse d'extinction due à l'oxygène;

$\left\{\mathrm{O}_{2}\right\}$ la concentration en oxygène de la solution considérée.
Nous avons mesuré, en présence d'oxygène, un temps de vie $\tau_{\mathrm{O}_{2}}$ de 1,18 ns (cf. Fig. $7 b$ ). Avec une concentration en oxygène $\left\{\mathrm{O}_{2}\right\}$ égale à $2 \times$ $10^{-3} \mathrm{M} / 1$ [13], on déduit pour $k_{\mathrm{O}_{2}}$ une valeur de $5 \times 10^{9} \mathrm{~s}^{-1} .1 / \mathrm{M}$ parfaitement compatible avec la valeur des constantes d'extinction de ce type de composé [9].

Notre système permet donc de mesurer sans problème insurmontable des durées de vie et des constantes de cinétique.

3.3 EVOLUTIONS SPECTRO-TEMPORELLES. - La figure 8 nous montre l'évolution spectrale au cours du temps du spectre de gain d'un composé organique polaire dissous dans un solvant polaire. On constate un glissement spectral important au cours des cent premières picosecondes suivant l'excitation. Ce glissement spectral montre une évolution temporelle du spectre de fluorescence, se traduisant par un élargissement inhomogène de ce spectre au cours du temps qui suit la création de cet état. Nous avons montré [14] que cet élargissement est dû à des effets d'environnement. La cage de solvant entourant la molécule, s'adapte à la conformation de l'état excité $S_{1}^{*}$, conformation différente de celle de l'état fondamental $S_{0}$ [14]. Ces résultats illustrent le fait que notre montage expérimental permet de résoudre, sur une échelle de quelques dizaines de picosecondes, des évolutions spectrotemporelles d'états excités.

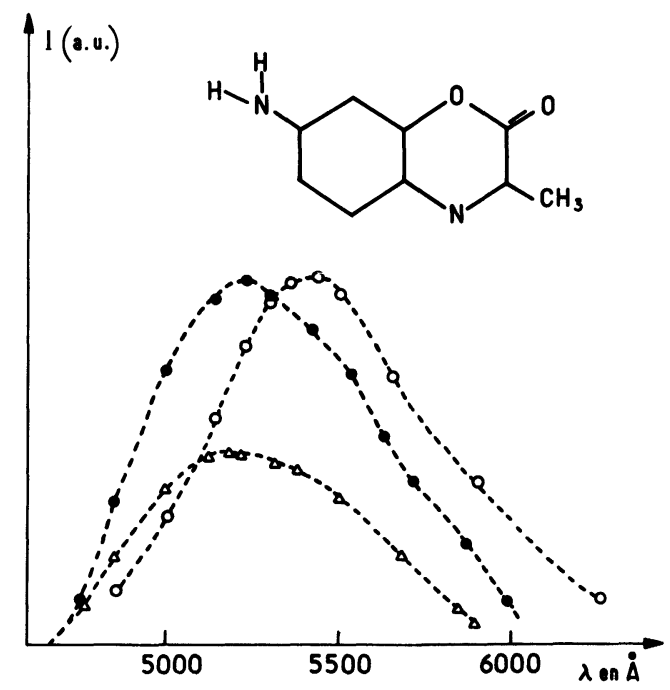

Fig. 8. - Spectre de gain en fonction du temps d'une solution d'amino-méthyl-benzoxazinone dans l'éthylèneglycol. $C=5 \times 10^{-5} \mathrm{M} / 1, T=20^{\circ} \mathrm{C} . \Delta: t=30 \mathrm{ps} ; \quad$ Q: $t=$ $50 \mathrm{ps} ;$ O:t $=350 \mathrm{ps}$.

[Study of amino-methyl-benzoxazinone in ethylene-glycol. Gain spectrum as a function of time. $C=5 \times 10^{-5} \mathrm{M} / \mathrm{l}$, $T=20^{\circ} \mathrm{C}, \Delta: t=30 \mathrm{ps} ; 0: t=50 \mathrm{ps} ; 0: t=350 \mathrm{ps}$.]

3.4 CinétiQue de RelaXation. - La figure 9 décrit la cinétique de croissance de la bande d'absorption $S_{1}^{*} \rightarrow S_{n}^{*}$ du perylène à $13600 \mathrm{~cm}^{-1}$ et de la bande d'amplification à $21200 \mathrm{~cm}^{-1}$. On constate que la 


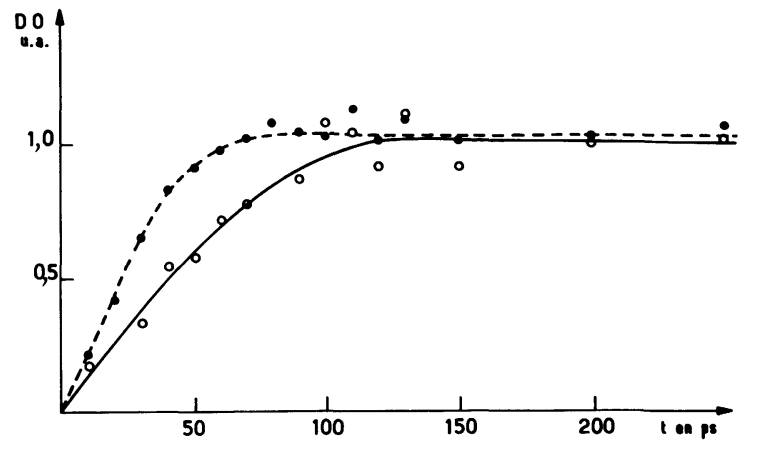

Fig. 9. - Cinétique de croissance de l'absorption et du gain à partir de l'état $S_{1}^{*}$ du perylène. Solution dans le cyclohexane. $C=10^{-4} \mathrm{M} / 1, T=20^{\circ} \mathrm{C}$. O croissance du gain à la fréquence $v=21200 \mathrm{~cm}^{-1}$; croissance de l'absorption à la fréquence $v=13600 \mathrm{~cm}^{-1}$.

[Time-increasing of absorption and gain from $S_{1}^{*}$ state of perylene. Solution cyclohexane. $C=10^{-4} \mathrm{M} / 1, T=20^{\circ} \mathrm{C}$. $O$ Increases of gain at frequency $v=21200 \mathrm{~cm}^{-1}$; Increases of absorption at frequency $v=13600 \mathrm{~cm}^{-1}$.]

bande d'amplification a un temps de croissance plus long que le temps de croissance de la bande d'absorption (environ 15 ps de différence). Le temps de croissance de la bande d'absorption est en fait contrôlé par la durée de l'impulsion. Ce temps de croissance constitue une mesure indirecte de la durée de l'impulsion excitatrice. Par contre, la croissance plus lente de la bande d'amplification illustre le fait que l'état qui amplifie efficacement la lumière n'est pas formé en même temps que l'impulsion. Dans notre montage expérimental, la longueur d'onde d'excitation utilisée pour le perylène est de $3530 \AA$. Lors de l'excitation on peuple en fait l'état $S_{2}^{*}$ ou des niveaux vibrationnellement excités $(v=1,2,3,4)$ de $S_{1}^{*}$. Après excitation, l'état $S_{2}^{*}$ ou les niveaux vibrationnels de $S_{1}^{*}$ relaxent vers l'état $v=0$ de $\mathrm{S}_{1}^{*}$. L'absorption de lumière peut avoir lieu à partir de tous ces états et la croissance de l'absorption est donc en phase avec l'impulsion qui crée ces niveaux. Par contre, pour des raisons de symétrie, l'amplification de lumière ne peut avoir lieu de façon efficace qu'à partir du niveau vibrationnel $v=0$ de l'état $\mathrm{S}_{1}^{*}$. L'amplification atteint son maximum lorsque tous les états créés ont relaxé vers l'état $v=0$ de $\mathrm{S}_{1}^{*}$, ce qui explique une croissance plus lente de l'amplification par rapport à la croissance de l'absorption. Ce retard constitue une mesure du temps de relaxation vibrationnelle de l'état $S_{2}^{*}$ vers l'état $S_{1}^{*}$ ainsi que des états vibrationnellement excités de $S_{1}^{*}$ [14]. Ces résultats montrent que notre dispositif expérimental permet d'étudier des cinétiques de croissance d'espèces excitées vers des échelles de temps de quelques dizaines de picosecondes.

\section{Conclusion.}

Nous avons montré qu'il est possible de concevoir un spectromètre d'absorption résolue dans le temps en n'utilisant qu'un seul faisceau d'analyse. Ceci permet alors d'utiliser comme détecteur une seule barrette de photodiodes et de profiter ainsi des avantages inhérents à ce type de détecteur (dynamique élevée, robustesse et faible coût). Dans ces conditions nous avons pu montrer par quelques exemples d'application que ce système était très performant. En particulier nous avons mesuré couramment des densités optiques de 0,01 . Des spectres d'absorption d'espèces excitées à durée de vie courte sont ainsi obtenus en quelques minutes entre 25000 et $10000 \mathrm{~cm}^{-1}$. Comme nous l'avons montré, cet appareillage a été appliqué avec succès à la mesure simultanée des spectres d'absorption et d'amplification d'états singulets excités de composés organiques, à la mesure de leur durée de vie ainsi qu'à l'étude de leur évolution spectrale résolue dans le temps.

\section{Remerciements.}

Nous tenons à remercier tout particulièrement $M$. le Professeur Ph. Kottis, Directeur de notre laboratoire pour l'intérêt constant qu'il a porté à la réalisation de ce montage, ainsi qu'à MM. M. Winckert et P. Lozano pour leur aide technique indispensable.

\section{Appendice $\mathbf{I}$.}

Nous allons montrer dans cet appendice qu'il est possible de mesurer $D O(v, t)$ en ne connaissant qu'une valeur homothétique de $I_{0}(v)$ et de $I(v, t)$.

En absence d'excitation la quantité $N_{\mathrm{S}^{*}}(t)$ est nulle et la densité optique $D O(v, t)$ est nulle quel que soit $v$ et $t$. Supposons maintenant qu'au lieu de mesurer réellement $I(v, t)$ nous mesurions une quantité $I^{\prime}(v, t)$ homothétique de $I(v, t)$ vérifiant la relation

$$
I^{\prime}(v, t)=K I(v, t) .
$$

Supposons que pour $I_{0}(v)$ nous obtenons aussi une relation semblable soit

$$
I_{0}^{\prime}(v)=K_{0} I_{0}(v) .
$$

Dans ce cas la densité optique apparente mesurée $D O^{\prime}(v, t)=\log \frac{I_{0}(v)}{I^{\prime}(v, t)}$ sera reliée à la densité optique réelle $D O(v, t)$ par la relation

$$
D O^{\prime}(v, t)=-\log K / K_{0}+D O(v, t) \text {. }
$$

Ceci est équivalent au déplacement de la ligne de base rencontrée dans les spectromètres d'absorption classiques. En absence d'excitation $\left(N_{\mathrm{S}^{*}}=0\right)$ la densité optique mesurée aura donc une valeur constante (quels que soient $v$ et $t$ ) différente de la valeur réelle nulle. En présence d'excitation $\left(N_{\mathbf{S}^{*}} \neq 0\right)$ la densité optique réelle $D O(v, t)$ sera alors obtenue de la façon suivante. Si le domaine spectral couvert est grand, il existe des fréquences $v_{0}$ pour lesquelles l'état $\mathrm{S}^{*}$ n'absorbe pas $\left(D O\left(v_{0}\right)=0\right)$. La densité optique apparente à ces fréquences est donc égale à $-\log K / K_{0}$; $D O(v)$ est alors donnée par l'expression suivante :

$$
D O(v) \doteq D O^{\prime}(v) \perp D O^{\prime}\left(v_{0}\right) .
$$




\section{Bibliographie}

[1] Greene, B. I., Hochstrasser, R. M. and Weisman, R. B., J. Chem. Phys. 70 (1979) 1247.

[2] Magde, D. and Windsor, M. W., Chem. Phys. Lett. 27 (1974) 31.

[3] Kobayashi, T. and Nagakura, S., Chem. Phys. Lett. 43 (1976) 429.

[4] Alfano, R. R. and Shapiro, S. L., Phys. Rev. Lett. 24 (1970) 592.

[5] Belke, S., Gase, R., Vogler, K., Opt. Quant. Elec. 12 (1980) 9.

[6] ShapIRo, S. L., Ultra short light pulses. Topics in Applied Physics (Springer Verlag, Berlin) 1977.

[7] BERLMANN, I. B., Handbook of fluorescence spectra of aromatic molecules (Academic Press, N.Y.) 1971.
[8] Goldschmidt, C. R. and Ottolenghi, M., J. Phys. Chem. 75 (1971) 3894.

[9] Founssier, J. P., Lougnot, D. J., Wieder, F. and FAURE, J., J. Photochem. 7 (1977) 17.

[10] Rullière, C., Laughrea, M. et Denariez-Roberge, M. M., Opt. Commun. 6 (1972) 407.

[11] Furomoto, H. and Ceccon, H., J. Appl. Phys. 40 (1969) 4204.

[12] Declemy, A., Rullière, C., Kottis, Ph., en préparation.

[13] MuncK, A. V. and ScotT, J. F., Nature 24 (1956) 587.

[14] Declemy, A., Rullikere, C., Kottis, Ph., en préparation. 